\title{
Epidemiological inferences using public information, influenza H7N9 epidemic in China
}

\author{
Eric H.Y. Lau*1, Jiandong Zheng², Tim K. Tsang1, Qiaohong Liao², Bryan Lewis ${ }^{3}$, John S. \\ Brownstein $^{4,5}$, Sharon Sanders ${ }^{6}$, Sumiko R. Mekaru4, Caitlin Rivers ${ }^{3}$, Gabriel M. Leung ${ }^{1}$, \\ Luzhao Feng ${ }^{2}$, Benjamin J. Cowling ${ }^{1}$ and Hongjie $\mathrm{Yu}^{2}$
}

${ }^{1}$ The University of Hong Kong, Hong Kong, Hong Kong; ${ }^{2}$ Chinese Center for Disease Control and Prevention, Beijing, China; ${ }^{3}$ Virginia Tech, Blacksburg, VA, USA; ${ }^{4}$ Boston Children's Hospital, Boston, MA, USA; ${ }^{5}$ Harvard Medical School, Boston, MA, USA; ${ }^{6}$ FluTrackers International Charity, Winter Park, FL, USA

\section{Objective}

This study described the strength and limitation of using line lists that built on publicly available data in various types of epidemiological inferences during the H7N9 epidemic in China, 2013.

\section{Introduction}

The influenza A(H7N9) virus emerged in early 2013 in China, with more than 130 laboratory-confirmed cases identified within a short period of about three months. Evidence-based public health response is essential for effective control of the disease, which relies on epidemiological and clinical data with good quality and timeliness. Publicly available information from sources such as official health website, online news, blogs or social media has the potential of rapid sharing of data to a wide community of experts for more comprehensive analyses. In our study we described the strength and limitation of these data for various types of epidemiological inferences.

\section{Methods}

We obtained historical archives of five line lists for influenza A(H7N9) cases based on publicly available information, created by Boston Children's Hospital (HealthMap), Virginia Polytechnic Institute and State University, FluTrackers, Bloomberg News and School of Public Health, University of Hong Kong, and also the official line lists from Chinese Center for Disease Control and Prevention, covering the period from early April to end of May. Demographic and epidemiological variables such as sex, age, province, city, date of illness onset, hospital admission, discharge, death and health status were available in most of the line lists.

We described the potential types of epidemiological inference that could be made for real-time severity and transmissibility assessment of the influenza A(H7N9) epidemic. Distributions of basic demographic and epidemiological variables were compared. We also compared the estimated onset-to-admission, onset-to-discharge and onset-to-death distributions and hospital fatality risk (HFR) inferred from different line lists, comparing to the official line list. Impact of live poultry market closure on H7N9 infection was also estimated in cities which implemented the intervention.

\section{Results}

Estimated age and sex distributions, epidemic curves, geographical spread, onset-to-hospitalization and onset-to-death distributions from line lists using public information were similar to those estimated from the official line list, at different time points over the course of the H7N9 epidemic. Live poultry market closure was found to be significantly associated with a lower H7N9 incidence in Shanghai, Nanjing and Hangzhou.

We estimated a shorter onset-to-discharge period from the line lists based on public information, compared to the official line list. The estimated HFRs from these line lists did not converge to the final estimate towards the end of the epidemic, when the outcomes of most hospitalized patients were available.

\section{Conclusions}

The results suggest that public information is able to provide accurate information on demographic characteristics and case counts. They also tend to report more detailed information for severe and death cases. However, information on patient status, hospitalization or discharge was less reliable. Publicly available information is likely to provide reliable information for inference of transmissibility or geographical spread but limited information on disease severity. It will be beneficial to public health by developing a protocol to setup an open-access minimum dataset based on publicly available data, with key epidemiological variables of standardized format and definition, along with a time stamp for each variable. This will allow a larger group of experts to carry out more timely and comprehensive epidemiological analyses when a novel disease emerges.

\section{Keywords}

Epidemiological inference; severity; transmissibility; line list; influenza H7N9

*Eric H.Y. Lau

E-mail: ehylau@hku.hk 